\title{
Second-line treatment for advanced NSCLC without actionable mutations: is immunotherapy the 'panacea' for all patients?
}

\author{
Alessandro Morabito
}

\begin{abstract}
The therapeutic approach for the second-line treatment of patients with advanced non-small cell lung cancer (NSCLC) without actionable mutations has been revolutionized by the recent approval of new effective drugs with various mechanisms of action, including nintedanib, ramucirumab, nivolumab, pembrolizumab, atezolizumab, and afatinib. The recent network meta-analysis of Créquit et al. (BMC Medicine, 15:193, 2017) compared the effectiveness and tolerability of the second-line treatments for advanced NSCLC with wild-type or unknown status for EGFR. The authors found that immunotherapy might be more efficacious than the currently recommended treatments. However, their meta-analysis does not take into account the role of predictive biomarkers - this is indeed a crucial point in the decision-making process considering that only a fraction of advanced NSCLC patients might derive a long-term benefit from second-line immunotherapy. The identification of molecular biomarkers that can predict a response to immune checkpoints, angiogenesis, and EGFR inhibitors remains an important goal of clinical research in order to maximize the benefit of these agents and to aid clinicians in the decision-making process.
\end{abstract}

Please see related article: https://bmcmedicine.biomedcentral.com/articles/10.1186/s12916-017-0954-x

Keywords: Nivolumab, Pembrolizumab, Atezolizumab, Afatinib, Nintedanib, Ramucirumab, Docetaxel, Pemetrexed, Erlotinib, NSCLC, Second-line therapy

\section{Background}

The recommended therapeutic options for the secondline treatment of patients with advanced non-small cell lung cancer (NSCLC) without actionable mutations has, until recently, mainly included docetaxel, pemetrexed (only for non-squamous histology), and erlotinib [1, 2]. This therapeutic approach has now been revolutionized by the approval of new effective drugs with various mechanisms of action, including angiogenesis, immune checkpoint, and EGFR inhibitors (Table 1) [3-9]. In patients with non-squamous histology, nintedanib or ramucirumab plus docetaxel, nivolumab, atezolizumab,

Correspondence: a.morabito@istitutotumori.na.it;

alessandromorabito1@virgilio.it

The Medical Oncology Unit, Thoracic-Pulmonary Department, Istituto Nazionale Tumori, "Fondazione G. Pascale" - IRCCS, Via Mariano Semola, 80131 Naples, Italy and pembrolizumab (in patients with programmed death ligand-1 (PD-L1) $>1 \%$ ) prolonged overall survival compared to docetaxel single agent $[3,4,6-8]$. In patients with squamous histology, ramucirumab plus docetaxel, nivolumab, atezolizumab, pembrolizumab (in patients with PD-L1 > 1\%), or afatinib were more efficacious than docetaxel or erlotinib [4, 5, 7-9]. Therefore, with the increasing number of available therapeutic options and patients approaching a second-line therapy, the therapeutic scenario has become more complex and the choice of the best second-line treatment is proving a significant challenge for oncologists.

\section{Network meta-analysis of second-line treatments}

In the network meta-analysis of Créquit et al. [10], the authors compared the effectiveness and tolerability of the second-line treatments for advanced NSCLC with 
Table 1 New approved drugs for the second-line treatment of patients with advanced NSCLC

\begin{tabular}{|c|c|c|c|c|c|c|c|}
\hline Reference & Patients & Histotype & Regimen & Response & $\begin{array}{l}\text { Progression-free } \\
\text { survival, months }\end{array}$ & $\begin{array}{l}\text { Median overall } \\
\text { survival, months }\end{array}$ & $P$ \\
\hline $\begin{array}{l}\text { Reck } \\
\text { et al. [3] }\end{array}$ & 658 & Adenocarcinoma & $\begin{array}{l}\text { Docetaxel + nintedanib vs. } \\
\text { docetaxel + placebo }\end{array}$ & $4.7 \%$ vs. $3.6 \%$ & 4.0 vs. 2.8 & 12.6 vs. 10.3 & 0.0359 \\
\hline $\begin{array}{l}\text { Garon } \\
\text { et al. [4] }\end{array}$ & 1253 & All histologies & $\begin{array}{l}\text { Docetaxel + ramucirumab vs. } \\
\text { docetaxel + placebo }\end{array}$ & $23 \%$ vs. $14 \%$ & 4.5 vs. 3.0 & 10.5 vs. 9.1 & 0.023 \\
\hline $\begin{array}{l}\text { Brahmer } \\
\text { et al [5] }\end{array}$ & 272 & Squamous & Nivolumab vs. docetaxel & $20 \%$ vs. $9 \%$ & 3.5 vs. 2.8 & 9.2 vs. 6.0 & $<0.001$ \\
\hline $\begin{array}{l}\text { Borghaei } \\
\text { et al. [6] }\end{array}$ & 582 & Adenocarcinoma & Nivolumab vs. docetaxel & $19 \%$ vs. $12 \%$ & 2.3 vs. 4.2 & 12.2 vs. 9.4 & 0.002 \\
\hline $\begin{array}{l}\text { Herbst } \\
\text { et al. [7] }\end{array}$ & 1034 & All histologies & $\begin{array}{l}\text { Pembrolizumab } 2 \text { mg/kg vs. } \\
\text { pembrolizumab } 10 \text { mg/kg vs. } \\
\text { docetaxel }\end{array}$ & $18 \%$ vs. $18 \%$ vs. $9 \%$ & 3.9 vs. 4.0 vs. 4.0 & 10.4 vs. 12.7 vs. 8.5 & $\begin{array}{l}0.0008 \\
<0.0001\end{array}$ \\
\hline $\begin{array}{l}\text { Rittmeyer } \\
\text { et al. [8] }\end{array}$ & 287 & All histologies & Atezolizumab vs. docetaxel & $14 \%$ vs. $13 \%$ & 2.8 vs. 4.0 & 13.8 vs. 9.6 & 0.0003 \\
\hline $\begin{array}{l}\text { Soria } \\
\text { et al. [9] }\end{array}$ & 795 & Squamous & Afatinib vs. erlotinib & $6 \%$ vs. $3 \%$ & 2.6 vs. 1.9 & 7.9 vs. 6.8 & 0.0077 \\
\hline
\end{tabular}

wild-type or unknown status for EGFR. Nivolumab, pembrolizumab, atezolizumab, and pemetrexed plus erlotinib were shown to be significantly more effective in terms of overall survival than docetaxel, pemetrexed, erlotinib, or gefitinib, and together with erlotinib plus cabozantinib represented the five most effective treatments in terms of overall survival. Indeed, the 'old' four recommended treatments were ranked in the 30th position, with no difference in effectiveness between them being observed. The authors' main conclusion was that immunotherapy is more efficacious than the current recommended treatments in the second-line treatment of patients with NSCLC without actionable mutations.

Nevertheless, a major limitation of this network metaanalysis was the inclusion of only a small number of trials designed in a population of patients selected for biomarkers. Therefore, the predictive role of biomarkers, which is indeed a crucial point in the decision-making process, was not considered. Currently, only a fraction of advanced NSCLC patients might derive a long-term benefit from second-line immunotherapy.

\section{Predictive biomarkers}

In patients with non-squamous histology, the CheckMate-057 study demonstrated a longer overall survival with nivolumab compared with single agent docetaxel (12.2 vs. 9.4 months, HR 0.73, $P=0.002)$, but patients with poorer prognostic factors and/or more aggressive disease combined with lower or no PD-L1 expression appeared to be at higher risk of death within the first 3 months on nivolumab versus docetaxel [11]. Exploratory analyses suggested that higher levels of PD-L1 were associated with a greater magnitude of overall survival benefit with nivolumab [12]. The role of PD-L1 as a predictive biomarker has also been demonstrated for pembrolizumab and atezolizumab, as confirmed by recent meta-analyses [7, 8, 13, 14]. However, there are a number of PD-L1 testing limitations that can confound its use as a predictive biomarker, including the heterogeneity and dynamics of PD-L1 expression, the varying performance of the various immunohistochemistry-based assays with different cutoffs, the absence of consensus regarding the relevance of geographic patterns of expression of PD-L1 or its expression on tumor or inflammatory cells within the tumor microenvironment and, finally, the availability of an adequate sample [15].

On the other hand, the LUME-Lung-1 study [3] showed that nintedanib (a triple angiokinase inhibitor) plus docetaxel significantly improved overall survival in pretreated patients with adenocarcinoma histology (12.6 vs. 10.3 months, HR $0.83, P=0.0359$ ), with a greater survival advantage in patients with early progression of disease or refractory to first-line therapy, or with a greater tumor burden, as well as a non-negligible number of 'long-surviving' patients (over 32 months).

Therefore, both molecular and clinical criteria should be considered in the decision-making tree of nonsquamous NSCLC patients, including PD-L1 expression and clinical factors associated with higher probability of response to nintedanib plus docetaxel (early progression or resistance to first-line therapy, high disease burden) or lower probability of response to nivolumab (progression disease as best response to prior treatment, early progression, five or more sites with lesions, bone or hepatic metastases, non-smoker status) (Fig. 1) [16, 17].

In patients with squamous histology, the CheckMate017 study demonstrated the superiority of nivolumab over docetaxel regardless of PD-L1 expression level [5]. However, in this setting, there are additional options of treatment represented by afatinib or ramucirumab plus docetaxel (Fig. 2). In the Lux-Lung-8 study [9, 18], 


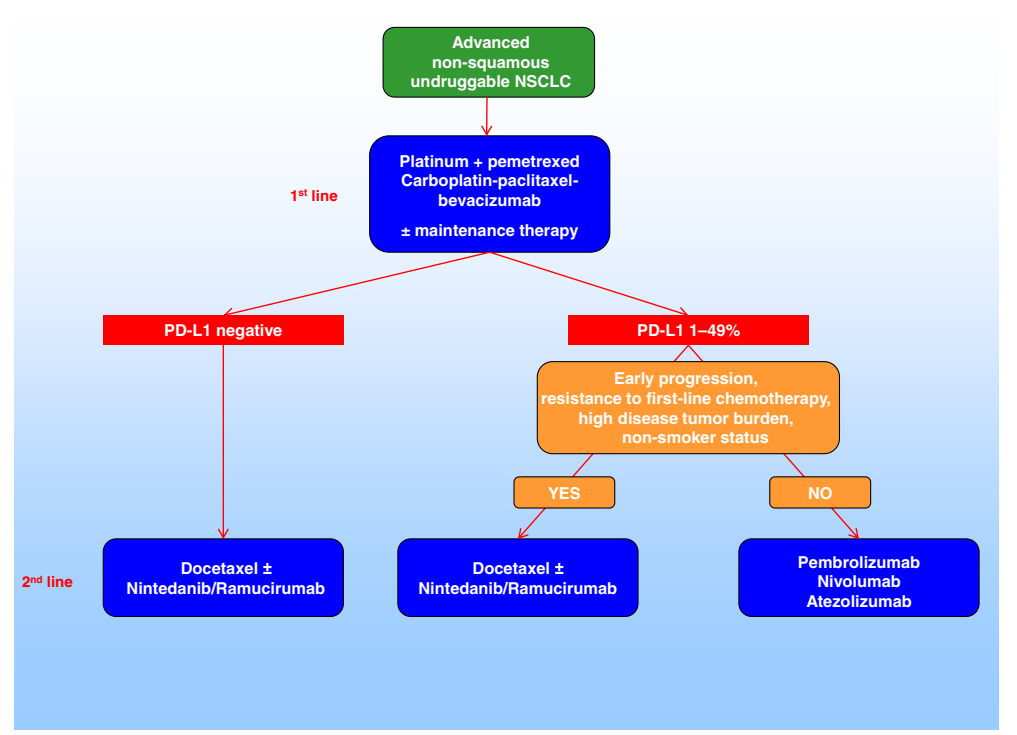

Fig. 1 Therapeutic scenario for patients with advanced non-squamous 'undruggable' NSCLC (EGFR wild type, ALK and ROS1 non-rearranged, PD-L1 < 50\%)

afatinib, an irreversible inhibitor of multiple members of the EGFR family, was superior to erlotinib, with $5 \%$ of patients being long-term responders (median overall survival of nearly 2 years). Exploratory analyses are ongoing to better define the molecular characteristics of patients associated to prolonged survival and, to date, a VeristratGood serum protein test and the presence of ErbB family mutations have been highlighted as potential biomarkers of long-term response to afatinib [19, 20]. In the REVEL study [4], ramucirumab, a totally humanized IgG1 monoclonal antibody that specifically targets the extracellular domain of VEGFR2, plus docetaxel was superior to docetaxel single agent, although it was associated with a worst toxicity profile. This is the first evidence supporting the use of an angiogenesis inhibitor also in patients with squamous histology. Unfortunately, to date, there are no validated biomarkers that could predict response to ramucirumab as well as nintedanib.

Therefore, the identification of proper predictive biomarkers for immunotherapy, angiogenesis, or EGFR inhibitors remains a crucial point in the era of precision medicine, and would likely contribute to the optimization of patient or treatment selection; this should be pursued in future studies.

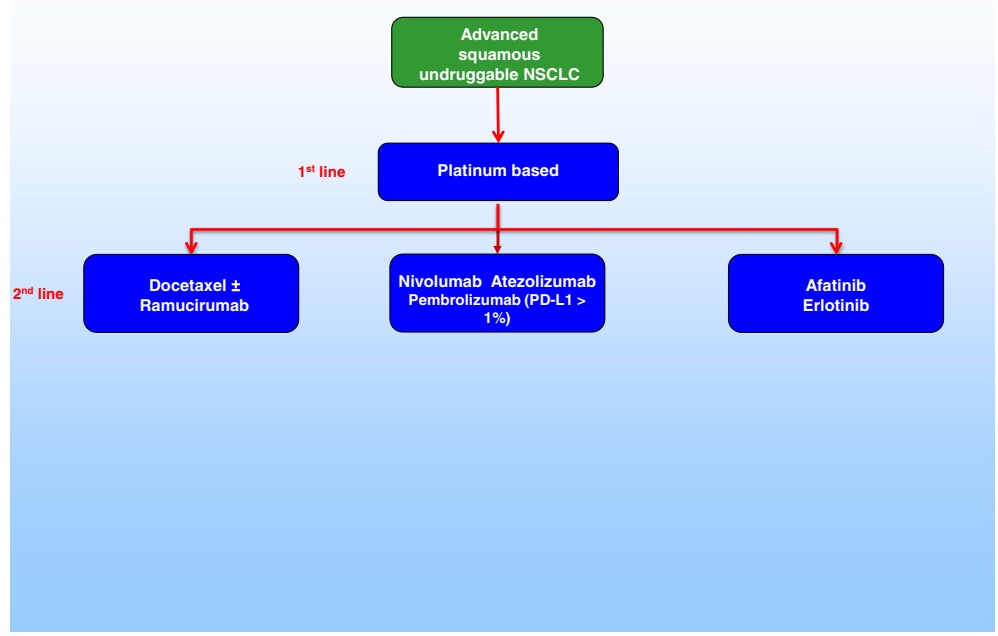

Fig. 2 Therapeutic scenario for patients with advanced squamous 'undruggable' NSCLC (PD-L1 < 50\%) 


\section{Future implications for clinical practice}

PD-L1, notwithstanding all its limitations, is to date the only molecular factor able to guide the choice of a second-line therapy for patients with advanced nonsquamous non-oncogene-addicted NSCLC. A number of additional factors are under investigation, including the tumor mutational burden, tumor-infiltrating lymphocytes, indoleamine 2,3-dioxygenase, DNA mismatch repair deficiency, and the expression of inflammatory genes. Moreover, by examining histological sections of tumor biopsies collected from patients prior to receiving immunotherapy, three basic immune profiles that correlate with response to anti-PD-L1/PD-1 therapy have been described, namely an immune-inflamed phenotype generally correlated with higher response rates to anti-PD-L1/PD-1 therapy, an immune-excluded phenotype associated with uncommon clinical responses, and an immune-desert phenotype rarely responsive to anti-PD-L1/PD-1 therapy [21]. Finally, emerging data also suggests that a subset of patients even appears to experience a tumor flare under checkpoint inhibitors, which has been recognized as a novel aggressive pattern of disease termed hyper-progression [22]. A hyper-progression can be observed in roughly $10 \%$ of immunotherapy-treated patients and specific genomic alterations, e.g., the presence of MDM2 family amplification or EGFR aberrations, seem to be associated with this clinical feature [23].

\section{Conclusions}

In conclusion, the network meta-analysis of Créquit et al. [10] showed that immunotherapy might be more efficacious than the current recommended treatments in the second-line therapy of NSCLC; nevertheless, immunotherapy cannot be considered the 'panacea' for all NSCLC patients.

Currently, both clinical and molecular criteria (to date by detection of PD-L1) should be considered in the definition of the best therapeutic approach of patients with pre-treated NSCLC without actionable mutations. In the future, genomic and immune profiles will help to identify patients eligible for immunotherapy.

\section{Funding}

The Medical Oncology Unit, Thoracic-Pulmonary Department of the Istituto Nazionale Tumori, "Fondazione G. Pascale" - IRCCS, Naples is partially supported by Associazione Italiana per la Ricerca sul Cancro (AIRC).

\section{Author contributions}

AM, the sole author of this article, read and approved the final manuscript.

\section{Author information}

Alessandro Morabito was born on September 1, 1967, in Naples, Italy. He received his M.D. degree in 1991 and specialty in Oncology in 1995 at the University Federico II of Naples. Dr Morabito has been the Director of the Medical Oncology Unit, Thoracic-Pulmonary Department of the Istituto Nazionale Tumori, "Fondazione G. Pascale" - IRCCS, Naples since 2010. His research fields of major interest during recent years are lung cancer, target based agents and immunotherapy. Dr. Morabito is an active member of the American Society of Clinical Oncology
(ASCO). He has published more than 200 articles in international journals, with an impact factor $>1400$ points, a citation index $>12,000$, and an $\mathrm{H}$ index of 43 (Google Scholar).

\section{Competing interests}

The author has participated in speakers bureaus from Pfizer, AstraZeneca, Roche, Boehringer, Bristol Mayer Squibb, Merck Sharp, and Dohme.

\section{Publisher's Note}

Springer Nature remains neutral with regard to jurisdictional claims in published maps and institutional affiliations.

Received: 2 January 2018 Accepted: 22 January 2018

Published online: 16 February 2018

References

1. Masters GA, Temin S, Azzoli CG, Giaccone G, Baker S, Brahmer JR, et al. Systemic therapy for stage IV non-small-cell lung cancer: American Society of Clinical Oncology clinical practice guideline update. J Clin Oncol. 2015; 33(30):3488-515.

2. Reck M, Popat S, Reinmuth N, De Ruysscher D, Kerr KM, Peters S, on behalf of the ESMO Guidelines Working Group. Metastatic non-small-cell lung cancer (NSCLC): ESMO Clinical Practice Guidelines for diagnosis, treatment and follow-up. Ann Oncol. 2014;25 Suppl 3:27-39.

3. Reck M, Kaiser R, Mellemgaard A, Douillard JY, Orlov S, Krzakowski M, et al. Docetaxel plus nintedanib versus docetaxel plus placebo in patients with previously treated non-small-cell lung cancer (LUME-Lung 1): a phase 3, double-blind, randomised controlled trial. Lancet Oncol. 2014;15(2):143-55.

4. Garon EB, Ciuleanu TE, Arrieta O, Prabhash K, Syrigos KN, Goksel T, et al. Ramucirumab plus docetaxel versus placebo plus docetaxel for second-line treatment of stage IV non-small-cell lung cancer after disease progression on platinum-based therapy (REVEL): a multicentre, double-blind, randomised phase 3 trial. Lancet. 2014;384(9944):665-73.

5. Brahmer J, Reckamp KL, Baas P, Crinò L, Eberhardt WE, Poddubskaya E, et al. Nivolumab versus docetaxel in advanced squamous-cell non-small-cell lung cancer. N Engl J Med. 2015;373(2):123-35.

6. Borghaei H, Paz-Ares L, Horn L, Spigel DR, Steins M, Ready NE, et al. Nivolumab versus docetaxel in advanced nonsquamous non-small-cell lung cancer. N Engl J Med. 2015;373(17):1627-39.

7. Herbst RS, Baas P, Kim DW, Felip E, Pérez-Gracia JL, Han JY, et al. Pembrolizumab versus docetaxel for previously treated, PD-L1 positive, advanced non-small-cell lung cancer (KEYNOTE-010): a randomised controlled trial. Lancet. 2016:387:1540-50.

8. Rittmeyer A, Barlesi F, Waterkamp D, Park K, Ciardiello F, von Pawel J, et al. Atezolizumab versus docetaxel in patients with previously treated nonsmall-cell lung cancer (OAK): a phase 3, open-label, multicenter randomised controlled trial. Lancet. 2017;389(10066):255-65.

9. Soria JC, Felip E, Cobo M, Lu S, Syrigos K, Lee KH, et al. Afatinib vs erlotinib as second-line treatment of patients with advanced squamous cell carcinoma of the lung (LUX-Lung 8): an open-label randomized controlled phase 3 trial. Lancet Oncol. 2015;16(8):897-907.

10. Créquit $P$, Chaimani A, Yavchitz A, Attiche N, Cadranel J, Trinquart L, et al. Comparative efficacy and safety of second-line treatments for advanced non-small-cell lung cancer with wild-type or unknown status for epidermal growth factor receptor: a systematic review and network meta-analyses. BMC Med. 2017;15:193.

11. Peters S, Cappuzzo F, Horn L, Paz-Ares L, Borghaei H, Barlesi F, et al. OA03. 05 Analysis of early survival in patients with advanced non-squamous NSCLC treated with nivolumab vs docetaxel in CheckMate 057. J Thoracic Oncol. 2017;12(1):S253

12. Horn L, Spigel DR, Vokes EE, Holgado E, Ready N, Steins M, et al. Nivolumab versus docetaxel in previously treated patients with advanced non-small-cel lung cancer: two-year outcomes from two randomized, open-label, phase III trials (CheckMate 017 and CheckMate 057). J Clin Oncol. 2017;35(35):3924-33. https://doi.org/10.1200/JCO.2017.74.3062.

13. Passiglia F, Bronte G, Bazan V, Natoli C, Rizzo S, Galvano A, et al. PD-L1 expression as predictive biomarker in patients with NSCLC: a pooled analysis. Oncotarget. 2016;7(15):19738-47.

14. Aguiar Jr PN, Santoro IL, Tadokoro H, de Lima LG, Filardi BA, Oliveira P, et al. The role of PD-L1 expression as a predictive biomarker in advanced non-smallcell lung cancer: a network meta-analysis. Immunotherapy. 2016;8(4):479-88. 
15. Griqq C, Rizvi NA. PD-L1 biomarker testing for non-small cell lung cancer: truth or fiction? J Immunother Cancer. 2016;4:48.

16. Popat S, Mellemgaard A, Reck M, Hastedt C, Griebsch I. Nintedanib plus docetaxel as second-line therapy in patients with non-small-cell lung cancer of adenocarcinoma histology: a network meta-analysis vs new therapeutic options. Future Oncol. 2017;13(13):1159-71.

17. Cortinovis D, Gregorc V, Migliorino MR, Abate MI, Manzo A, Malapelle U, Morabito A. New perspectives in the second-line treatment of non squamous NSCLC patients: results from a large Italian Lung Cancer Working Group. Crit Rev Oncol Hematol. 2017;109:35-41.

18. Giordano P, Manzo A, Montanino A, Costanzo R, Sandomenico C, Piccirillo MC, et al. Afatinib: an overview of its clinical development in non-small-cell lung cancer and other tumors. Crit Rev Oncol Hematol. 2016;97:143-51.

19. Gadgeel S, Goss G, Soria JC, Felip E, Georgoulias V, Lu S, et al. Evaluation of the VeriStrat ${ }^{\oplus}$ serum protein test in patients with advanced squamous cell carcinoma of the lung treated with second-line afatinib or erlotinib in the phase III LUX-Lung 8 study. Lung Cancer. 2017;109:101-8.

20. Goss G, Felip E, Cobo M, Lu S, Syrigos K, Lee KH, et al. P3.01-043 Impact of ErbB mutations on clinical outcomes in afatinib- or erlotinib-treated patients with SCC of the lung. J Thoraic Oncol. 2017;12(11 Suppl 2):S2217-18.

21. Chen DS, Mellman I. Elements of cancer immunity and the cancer-immune set point. Nature. 2017;54(7637):321-30.

22. Champiat S, Dercle L, Ammari S, Massard C, Hollebecque A, Postel-Vinay S, et al. Hyperprogressive disease is a new pattern of progression in cancer patients treated with anti-PD-1/PD-L1. Clin Cancer Res. 2017;23(8):1920-8.

23. Kato S, Goodman A, Walavalkar V Barkauskas DA, Sharabi A, Kurzrock R. Hyperprogressors after immunotherapy: analysis of genomic alterations associated with accelerated growth rate. Clin Cancer Res. 2017;23(15):4242-50.

\section{Submit your next manuscript to BioMed Central and we will help you at every step:}

- We accept pre-submission inquiries

- Our selector tool helps you to find the most relevant journal

- We provide round the clock customer support

- Convenient online submission

- Thorough peer review

- Inclusion in PubMed and all major indexing services

- Maximum visibility for your research

Submit your manuscript at www.biomedcentral.com/submit

) Biomed Central 